\title{
Reliable wireless MAC layer for industrial internet of things using polarisation diversity
}

\author{
Ritesh Kumar Kalle \\ Hitachi India Pvt. Ltd., \\ Unit No. S 704, 7th floor, \\ World Trade Center, No. 26/1, \\ Malleswaram, Bangalore, 560-055, India \\ Email: ritesh@hitachi.co.in
}

\begin{abstract}
Industrial internet of things (IIoT) requires superior reliability from underlying communication technology that consumer wireless technologies often fail to meet. Utilising polarisation diversity techniques through rotating polarisation waves (RPWs) have been recently proposed to improve the coverage and reliability of IIoT network. However, the design of medium access (MAC) layer that optimally utilises the key features of the underlying physical layer is a key challenge especially when considering multihop networks. Adopting conventional MAC protocols based on carrier sensing or time division alone would not utilise the physical layer in its optimal manner leading to lower throughput and higher end-to-end latency. In this paper, novel medium access techniques to improve the reliability in the RPW wireless communication have been described. We also consider extensions to the MAC layer to support multihop network using the tree topology. An overview of MAC layer methods and comparative mathematical analysis is provided that clearly show the benefits of multi-hop RPW over conventional networks.
\end{abstract}

Keywords: IoT; reliable wireless network; polarisation diversity; orthogonal code; packet combination; priority; multihop; tree topology; transmission airtime.

Reference to this paper should be made as follows: Kalle, R.K. (2018) 'Reliable wireless MAC layer for industrial internet of things using polarisation diversity', Int. J. Digital Enterprise Technology, Vol. 1, Nos. 1/2, pp.96-120.

Biographical notes: Ritesh Kumar Kalle received his $\mathrm{PhD}$ in Wireless Networks from the International Institute of Information Technology, Bangalore in 2014. He is currently a Senior Researcher at Hitachi India R\&D Centre. His research interests include wireless technologies for IoT, energy efficiency, reliability and QoS at protocol layers. He is a senior member of IEEE and ballot group member of several IEEE standards.

This paper is a revised and expanded version of a paper entitled 'Reliable and prioritised communication using polarisation diversity for Industrial Internet of Things', presented at ICWISE 2016, Malaysia, 11 October 2016. 


\section{Introduction}

Industrial internet of things (IIoT) is a special class of industrial networks with the objective of interconnecting sensors embedded in the infrastructure with monitoring systems deployed in the field or hosted on cloud services. The information and control signal flow in both directions and hardware resources are expected to perform reliably for extended periods (several years without maintenance or replacement). Most consumer grade wireless technologies do not qualify these requirements and therefore new radio technologies are being investigated to meet industrial requirements.

For reliability in IIoT scenario, the principle of spatial and spectral redundancy is widely applied to wireless communication. Spatial redundancy depends on physical or link layer schemes to allow each node to communicate with multiple neighbours, such that the routing schemes at the network layer may use multiple routes to the same destination of information. Spectral variant of redundancy makes use of multiple channels in the radio frequency (RF) spectrum. Combined with medium access (MAC) protocol, this feature provides the flexibility of using redundant channels or hopping amongst available channels to minimise the impact of interference.

A comprehensive survey of standards and technologies for wireless adoption in industrial applications is provided in Wang and Jiang (2016). Their study reveals that data reliability is single largest deciding factor in the adoption of wireless technology in industries $(95.5 \%)$. Three major issues impede the growth of industrial wireless IoT technologies:

1 The first issue is the lack of a convergence solution for these standards. A common standard can lower risk associated with the infrastructure investment, and then stimulate the widespread industrial adoption.

2 The second issue is the lack of detailed performance evaluation of the systems in industrial environment. Benchmarking experiments in controlled environments would help to measure the strengths and shortcomings of these systems.

3 The third issue to be resolved for wider deployment of wireless networks in industry is the lack of effective evaluation and diagnostic platforms for industrial wireless systems such as those utilised for cellular and Wi-Fi.

In Nobre et al. (2015), the authors survey the higher layer protocols for routing and scheduling data over the wireless sensor network such as WirelessHART. The study indicates that monitoring applications can use mesh networks to improve their reliability using spatial redundancy (multiple paths), and control applications can use temporal redundancy (multiple copies of the transmitted data). However, current standards do not support the adaptability of protocols suited to each application.

A detailed survey of communication and networking technologies has been provided in Rüth et al. (2017). The survey includes both legacy wired networks as well as industrial IoT standards such as ISA 100.11a and describe that critical control applications have not been enabled with wireless networks due to their highly variable reliability performance. The paper also describes the MAC and network layer challenges impacting the performance of networks in factory applications. 
Liu et al. (2014) describe a TDMA-based approach to improve the reliability, synchronisation time and reduce latency in a multi access point environment. The approach is also implemented in hardware and performance is evaluated in test conditions.

Marchenko et al. (2014) extend the scenario to include relay nodes that improve the range and signal quality of wireless transmissions. This work presents the results of an experimental study of selective cooperative relaying protocols that are implemented in off-the-shelf IEEE 802.15.4-compatible devices and evaluated in an industrial production plant. Periodic, adaptive, and reactive relay selection methods are evaluated and results show that they outperform the conventional time diversity retransmissions. However, the study is more experiment oriented and no mathematical model is provided, that captures the benefits introduced through selective cooperative relay mechanism.

Lack of deployment time validation procedures in IoT networks has been analysed in detail in Todolí-Ferrandis et al. (2017) and motivated the requirement of deployment time protocol to optimise the path quality in addition to the active network adaptation of routes to meet requirements of the industrial applications. The authors propose a quick deploy sensor network (QDSN) procedure during deployment that measures additional variables related to path quality such as link, delivery rate, round trip time, neighbouring nodes, etc.) The deployment is then fine tuned to meet the acceptable performance.

Hassan et al. (2017) evaluate the application of WirelessHART for real time control applications. Through a simulation driven study, it is revealed that latency and packet losses are two most important challenges of the network when applied to control applications. Moreover, conventional networked control algorithms presume input/output paths to be reliable and measurements are received periodically. Packet losses in wireless environment accumulate error based on the last received value leading to a possibility of large process oscillations once the communication is restored. Therefore, lower layer techniques to mask the channel error and maintain acceptable network reliability is an important feature for industrial IoT applications.

Conventional means have benefited the system throughput and reliability of low power wireless access networks to certain extent, but has often inadequately addressed the problem of dead-points in communication due to multipath introduced by electromagnetic reflective surfaces in the vicinity such as those illustrated in Matsuzaki (2012). Especially during quasi-static scenario, prolonged deep fades may occur at certain positions in the industrial deployment. Such situations arise for example in a manufacturing setup, which generally have low relative motion and quasi-static channels. However, whenever equipment setup is changed or moved, small number of sensors may enter long periods of high attenuation due to reflections and multipath.

In the context of IIoT networks, new radio wave propagation mode for sub-GHz bands using practical circuits and low-cost infrastructure has been proposed in literature such as Takei (2013) and Takei (2014a). It is also analytically established that the received RF power and therefore the bit error rate (BER) of rotating polarisation wave (RPW) in rich scattering environment in presence of large metallic scatterers is always better than the conventional Rayleigh faded channel as shown in Takei (2014b).. This technology is suitable for quasi-static or low mobility industrial environments such as factory floors, electricity sub-stations, elevators and wind power generators. Furthermore, use of sub-GHz unlicensed wireless frequency bands provides advantage of better coverage and lower packet drop rates. 
In this paper, we mainly focus on the star and multihop tree topology of RPW network from a protocol layer perspective because these two are the most preferred topologies in industrial wireless networks for factory and process automation. More specifically, the aspects considered in this paper relate to the MAC layer to support reliable and prioritised communication over an RPW system. This paper is organised as follows: Section 2 provides an overview of the RPW technology and outlines the key features. In Section 3, MAC layer scheme to support reliable and prioritised M2M communication using RPW is described. In Section 4, extension of MAC layer to support multihop tree topology using RPW is presented. A parsimonious model to describe the advantage of proposed MAC scheme is further presented. Finally, the conclusions are presented in the Section 5.

\section{RPW communication}

Figure 1 depicts the concept of RPW in industrial deployment. It is well known in electromagnetic theory that the polarisation of transmit and receive antenna plays an important role in determining the efficiency of signal energy transfer. Orthogonal polarisations at transmit and receive antenna positions result in minimum energy transfer. Thus conceptually, it would be beneficial to design a system where the polarisation of transmit antenna rotates slowly resulting in the expansion of codes in a manner such that the receiver be able to distinguish one of the original paths in the communication system. In this manner, it is possible to distinguish unique path, which may undergo different BERs. By selecting the path, that provides the best signal quality or otherwise by combining multiple copies of the frames sent over independent paths, a highly reliable low rate communication system can be achieved.

Figure 1 Conceptual representation of RPW in industrial deployment (see online version for colours)

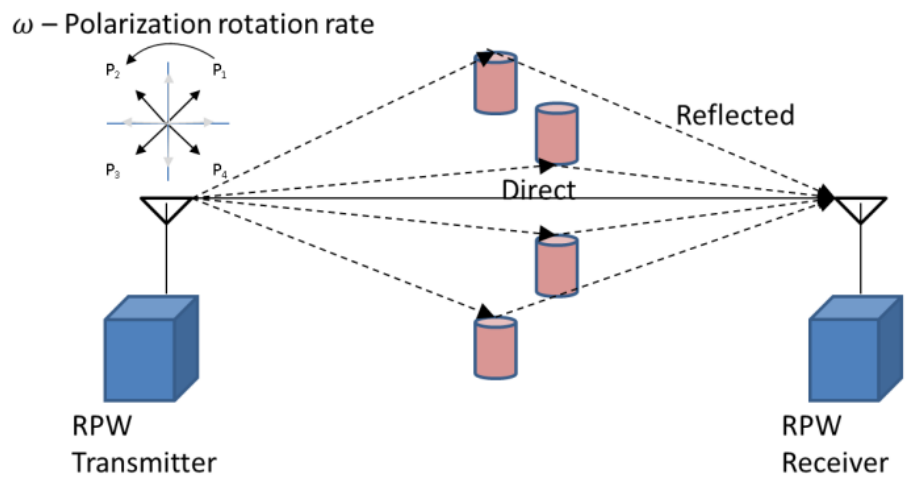

a Generation

$$
\begin{aligned}
Y_{v}(t) & =x(t)\left[\cos \left(2 \pi f_{1} t\right)-\cos \left(2 \pi f_{2} t\right)\right] \\
& =2 x(t)\left[\sin \left(2 \pi \frac{\delta_{1}}{2} t\right) \cdot \sin \left(2 \pi \frac{\delta_{2}}{2} t\right)\right]
\end{aligned}
$$




$$
\begin{aligned}
Y_{h}(t) & =x(t)\left[\sin \left(2 \pi f_{1} t\right)+\sin \left(2 \pi f_{2} t\right)\right] \\
& =2 x(t)\left[\sin \left(2 \pi \frac{\delta_{1}}{2} t\right) \cdot \cos \left(2 \pi \frac{\delta_{2}}{2} t\right)\right]
\end{aligned}
$$

where $Y_{v}(t)$ and $Y_{h}(t)$ are the outputs of the vertical and horizontal antennas respectively and the frequencies $\delta_{1} / 2$ and $\delta_{2} / 2$ correspond to the carrier frequency and the polarisation rotating frequency respectively. $x(t)$ is the chip sequence.

b Propagation

The RPW changes the direction of its polarisation-rotation when it is reflected from a reflector surface. The sum of waves arriving at a receiver consists of right and left handed RPWs as these waves experience reflections of odd or even numbers. The differentiation of polarisation can be achieved using (semi) orthogonal codes both at the transmitter and receiver as shown in Takei (2014a). Introduction of artificial fading have been shown to provide gains while scheduling across multiple users Viswanath et al. (2002). Through RPW, periodic opportunities of better channel conditions can be provided for node(s) stuck in deep fade condition. However, efficient multi-user scheduling algorithm in RPW scheme to achieve this gain remains to be designed.

c Reception

To obtain a receiving wave on an arbitrary polarisation, the receiver uses two spatially perpendicular antennas. The outputs from the two antennas are amplified and down-converted using the local carrier frequency (equal to the average of two carriers of RPW) and the intermediate frequency. This signal is later decoded by block codes to recover the information. There are several advantageous effects of using the polarisation diversity for transmission and reception of information in industrial environments with multiple reflectors. This includes greater control in exploiting the path diversity using the phase angle approach, avoiding situations where receivers are permanently in deep fade condition, etc. However, challenges exist with respect to achieving accurate frame synchronisation, stable operational $\operatorname{BER}\left(<10^{-5}\right)$ conditions, etc. Also, the practical data rates achieved in prototypes are less than $100 \mathrm{kbps}$. This motivates the design of low overhead, high reliability link layer protocols.

\section{MAC layer for reliable communication in industrial environment}

\subsection{Polling-based MAC for industrial applications}

Standards for wireless communications such as IEEE 802.11 and IEEE 802.15.4 specify random access-based multiple access schemes termed carrier sensing multiple access with collision avoidance (CSMA/CA). CSMA provides higher channel utilisation, 
however jitter and higher average latencies for data transmission are important challenges for industrial applications. Amendments to these standards that support contention free access periods also make use of polling to determine the order of client transmissions and length of each slot. These exhaustive polling schemes however do not guarantee latencies. Proprietary modifications to these standards introduce selective polling periods to dynamically adapt the scheduling order (Fujiwara et al., 2014). However, these MAC protocols cannot be directly applied to the RPW communication system due to the continuous change induced in the channel conditions. To this end, a polling-based MAC protocol developed specifically for RPW system is proposed. Primary topologies for RPW communication network include the point-to-point and the star for multiple access scenarios. Figure 2 illustrates a typical cycle of polling and response in a Mono-Master star topology network of RPW nodes.

Abbreviations and important terms used in this discussion have been described in Table 1 for easy reference:

Table 1 Abbreviations and definitions used

\begin{tabular}{|c|c|}
\hline Terms & Description \\
\hline SHR & Synchronisation header \\
\hline PHR & Physical layer header \\
\hline MHR & Medium access control header \\
\hline SF-spec & Super frame specification: order, interval of beacons \\
\hline FCS & Frame check sequence ( 2 byte $\mathrm{CRC}$ code) \\
\hline $\mathrm{ACK}$ & Acknowledgement \\
\hline $\mathrm{CRC}$ & Cyclic redundancy check \\
\hline$t_{s f}$ & Super frame time \\
\hline$t_{b}$ & Beacon frame time \\
\hline$t_{\text {sifs }}$ & Inter frame time for master to slave switching \\
\hline$t_{m i f s}$ & Inter frame time for slave to master switching \\
\hline$T_{u}$ & Update period of a slave node, random variable \\
\hline$t_{u}, \sigma_{T_{u}}^{2}$ & Mean, variance of the update period \\
\hline$D$ & Alarm latency between generation time and successful transmission to the master \\
\hline$L$ & Length of packets in bits \\
\hline$T_{c}$ & Cycle time elapsed between two successive polls \\
\hline$B_{i}$ & i.i.d random variables that account for the times to transmit the data. \\
\hline$P_{\text {alarm }}$ & Probability that any slave has an alarm \\
\hline$T_{a c k}$ & Transmission time of a packet sent with acknowledgement in $k$ attempts \\
\hline$t_{a c k}, \sigma_{T_{a c k}}^{2}$ & Mean, variance of the transmission period \\
\hline$T_{\text {data }}$ & Wireless frame transmission time, ignoring failure \\
\hline$T_{\text {slot }}$ & Unit time spent in preparing a transmission \\
\hline
\end{tabular}


Figure 2 Illustration of one cycle of master poll and response from a slave (see online version for colours)

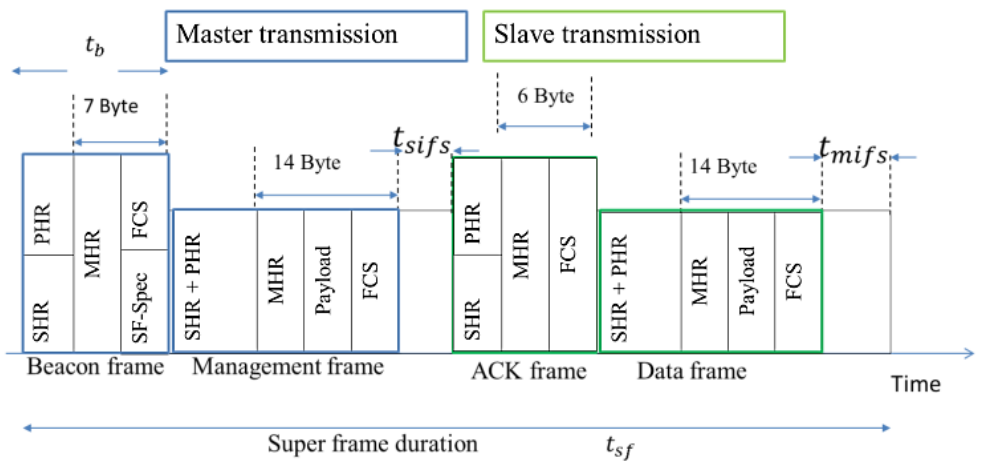

There are four main types of MAC frames in the RPW communication system, distinguished by special frame control header fields: beacon frame, data frame, ACK frame and Management frame. The lengths (bytes) of each frame types are currently optimised for low throughput RPW nodes. The role of a beacon frame transmission in the start of every super-frame is:

a synchronisation of other RPW nodes

b identification of master

c structure description of the super-frame.

Optionally, the beacon frame from the master and the ACK frame from the slave nodes can be transmitted at higher average power or at different periodicity for better synchronisation. The synchronisation code in the SHR is specially determined for the RPW network as in Perumal et al. (2016) and can also be used to select individual slave nodes if orthogonal codes are utilised (Takei, 2014a; Aono and Takei, 2014). The super-frame level synchronisation is achieved through latching on to special beacon frames at the beginning of each slot $\left(t_{s f}\right)$. Each beacon itself is aligned with the beginning of the superframe slot and occupies a fixed time $\left(t_{b}\right)$. The management and data frame can however have variable payloads.

The reciprocity and correlation of wireless channels in RPW system is yet to be established as shown in Perumal et al. (2016) and therefore the synchronisation and channel estimation on both the uplink and downlink transmissions is performed. The rate of polarisation rotation is matched to the bit transmission duration; thus, the synchronisation code also enables channel estimation and the ACK frame can also serve as a feedback channel. The order of polling the slave nodes and data transmission is determined by the polarisation channel controller and scheduler in the master node.

\subsection{Reliable communication using polarisation diversity}

Packet-level diversity at MAC layer complements the use of radio diversity techniques in the physical layer. RPW natively exploits diversity owing to rotation of polarisation that produces different realisations of wireless channel at each polarisation angle. By combining copies of packets transmitted at different polarisation angles in the MAC 
protocol, a more reliable data transmission can be achieved. In this section, we study the various MAC layer packet-combining methods proposed for conventional networks to improve the reliability of reception. Further, we identify the most common shortcoming of these methods, which is the existence of a hidden error scenario. Moreover, we propose a packet combining scheme for RPW network that overcomes the hidden error problem of conventional networks and thereby enhances the reliability of reception.

Figure 3 Packet selection and error classification algorithm

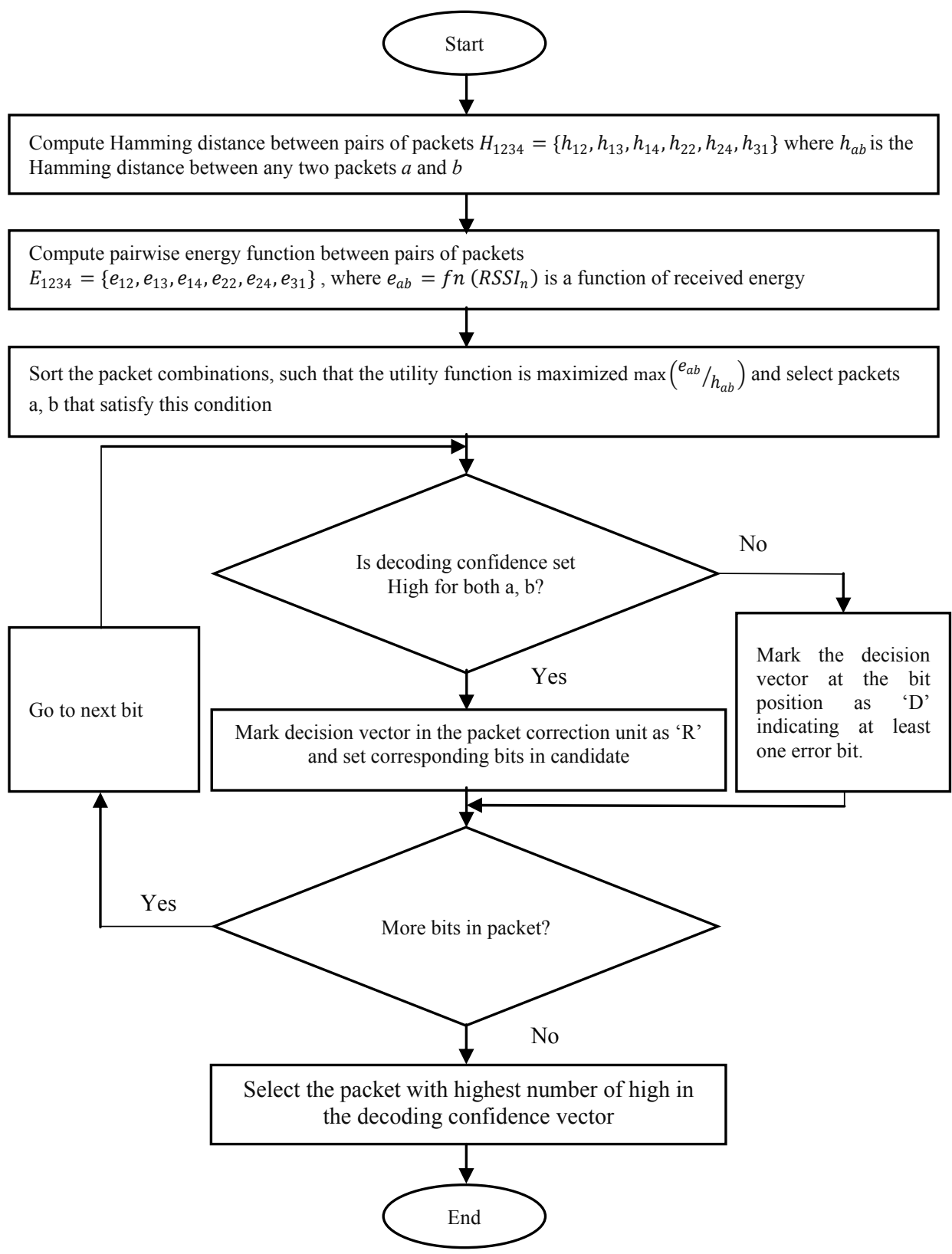


In literature, the following techniques are proposed for conventional wireless networks:

1 Diversity packet combining extended ARQ (EARQ) described in Viswanath et al. (2002) protocol combines erroneous copies of the same packet using bitwise logical XORs to locate errors in a combined copy. A brute force search method with CRC computation at every step is used to recover the correct copy. EARQ does not require coherent detection. Even if all the blocks received over the diversity branches are corrupt, they may be combined to retrieve the correct packet. However, the latency is high due to retransmissions and extensive CRC evaluation.

2 Simple packet combining (SPaC) proposed by Dubois-Ferrire et al. (2005) exploits the broadcast medium, spatial diversity of a multi-hop wireless network by using overheard packets at any node, in addition to the next-hop destination of the packet. If these corrupt copies correspond to identical transmissions of the same packet, then combining them corresponds to decoding a repetition code. In a multi-hop low rate wireless network, overheard packets can be combined either having the same or redundant information to improve the error recovery.

3 Packet merging proposed by O'Rourke and Brennan (2012) creates a merged error mask also known as 'ambiguity vector' $\left(\mathrm{V}_{\mathrm{A}}\right)$ at the destination by accepting two packets of the same type. It is then used with one of the packets, and the FCS to obtain the original packet.

This is achieved by modifying each bit position where there is a potential error (as indicated by $\mathrm{V}_{\mathrm{A}}$ ), and checking whether the resulting packet matches the FCS. Method to select the packet and the algorithm to search through bit combinations has been proposed.

However, none of the above conventional techniques are applicable to RPW communication system as the polarisation rotation spreads each information bit over multiple polarisation channels without the need of separate packet transmissions. Further, the retrieval process described in literature fails if there is at least one-bit position where both copies have an error (hidden error). The probability of occurrence of hidden error increases with rise in BER, thereby causing a downgrade in recovery speed. A six-step procedure is described which combines the packets decoded in multiple polarisation angles to reduce the error probability.

Step 1 consists of reception of the multiple packet copies decoded at distinct polarisation angles (Figure 1 depicts four such copies) and passed on to the MAC layer packet processor. Note that appropriate sampling at receiver at each polarisation angle is sufficient to generate these multiple copies. The packet reconstruction is only required when none of the multiple copies of packets received are deemed to be correct.

In Step 2, a candidate packet is derived through bit wise majority voting-based packet combining technique. The candidate packet is stored in a packet buffer that holds the estimate of the final packet derived through majority vote mechanism. Majority voting for even number of packet copies is inconclusive when equal number of $0 \mathrm{~s}$ and $1 \mathrm{~s}$ are found at the same position in the packets to be combined or the number of error bits is more than the correct bits at the same position. Step 5 recovers such errors.

Step 3 computes the subset of candidate packet where no decision could be made. It also selects two most probable packets amongst the multiple copies by packet selection algorithm described in Figure 3. This algorithm first computes the pairwise Hamming distance $\left(h_{a b}\right)$ for each of the packet copies. It also computes the pairwise function $\left(e_{a b}\right)$ of the received packet's energy profile. This can be based on the received signal strength 
indicator (RSSI) signal profile (correlation characteristics, weighted average, etc.). By maximising the utility function $\left(\max \left(e_{a b} / h_{a b}\right)\right)$, a pair of packets that are nearest to each other and having the highest received energy can be selected.

In Step 4, bit positions in the two probable packets are marked as:

1 recovered $(\mathrm{R})$ if decision made and assigned to candidate packet

2 common $(\mathrm{C})$ if bits match amongst the packets

3 different (D) if bits don't match. Both (C) and (D) positions indicate the chance of hidden error.

Step 5 isolates common (C) positions and classifies into jointly correct or error bits (hidden errors that cannot be identified in both packets) based on classification algorithm described in Figure 3. Decoding confidence metric is fed to the packet processor as cross layer information from the physical layer. The bit position with High decoding confidence is marked as ' 1 ' and those with Low decoding confidence is marked as ' 0 '. Positions where the one or both packets have low decoding confidence, a decision is not made and the bit position is marked ' $\mathrm{D}$ '. Decision is made at (C) marked positions with high decoding confidence in both copies and corresponding bits substituted in candidate packet. The packet candidate with highest number of high decoding confidence bits is utilised for the next step.

In a hard decision block decoder for RPW system, the recovered codeword is mapped to nearest valid codeword. A threshold-based classification (as high or low) of the Hamming distance between the two can serve as one of the decoding confidence metric. In case of soft decoding, an analogous metric may be computed based on correlation between the received samples $(Y)$ and each codeword $\left(C_{i}\right)$.

$$
C\left(Y, C_{i}\right)=\sum_{j=1}^{n} y_{j} c_{i j} .
$$

In Step 6, bit combinations of the selected packet (or sub-packet) are successively evaluated to satisfy the FCS. The packet is decoded to be the right packet if the FCS check is satisfied and an ACK is transmitted back. The packet candidates are evaluated in the order of increasing Hamming distance from the initial candidate packet. Also, the order of bit evaluation is dependent on pool of bit positions having low decoding confidence vector first and later with high decoding confidence vector. In case the FCS is satisfied by one of the combinations, the evaluation is terminated.

\section{Analysis}

Let $p_{e}$ denote the operating BER of the wireless link. The packet error rate (PER) can therefore be expressed as $p_{e}=1-\left(1-p_{e}\right)^{n}$ where $n$ is the packet size. Consider the combination of at least two erroneous packets to generate a candidate packet without our proposed algorithm. The procedure would fail, when at least one position in which both copies have error - hidden error scenario. Further, this procedure would fail if additional copies of packets also contain one or more hidden errors. The probability of success in at the reception of $L^{\text {th }}$ packet copy would be: 


$$
P(L)=\left[1-\sum_{r=1}^{L-1} P(r)\right] \cdot\left[\left(1-p_{e}\right)^{n}+\left(1-\left(1-p_{e}\right)^{n}\right)\left(1-\alpha_{d}(L)\right)\right]
$$

where $\alpha_{d}(L)$, is the probability of hidden error with all preceding $L-1$ copies in the packet buffer. In Chakraborty et al. (1999), it is shown,

$$
\alpha_{d}(L)=\frac{\sum_{K_{1}=1}^{n} \ldots \sum_{K_{L}=1}^{n} P\left(K_{1} \ldots K_{L}\right) \prod_{1 \leq i, j \leq L, i<j} P_{i, j}}{\left(1-\left(1-p_{e}\right)^{n}\right)^{L} \prod_{l=2}^{L-1} \alpha_{d}(l)}
$$

where, based on assumption independent occurrence of errors

$$
P\left(K_{1} \ldots K_{L}\right)=P\left(K_{1}\right) \cdot P\left(K_{2}\right) \ldots P\left(K_{L}\right)
$$

is the joint probability of the $L$ copies having $K_{1} \ldots K_{L}$ errors respectively.

$$
P\left(K_{i}\right)=\left(\begin{array}{c}
n \\
K_{i}
\end{array}\right) p_{e}^{K_{i}}\left(1-p_{e}\right)^{n-K_{i}} \text { being the probability of a copy having } K_{i} \text { error. }
$$

Further, $P_{i, j}$ is the probability that two copies with $K_{i}$ and $K_{j}$ errors, have a hidden error:

$$
P_{i, j}=1-\frac{\left(n-K_{i}\right) !\left(n-K_{j}\right) !}{n !\left(n-K_{i}-K_{j}\right) !} .
$$

It can be considered that, the proposed algorithm in this paper eliminates the probability of occurrence of hidden error $\left(\alpha_{d}(L) \rightarrow 0\right)$. Therefore from equation (4),

$$
P_{\text {proposed }}(L)=\left[1-\sum_{r=1}^{L-1} P(r)\right] \text {. }
$$

The relative benefit in terms of decoding success probability can therefore be expressed in percentage as

$$
\left(P_{\text {proposed }}(L)-P(L)\right) * 100 / P_{\text {proposed }}(L) \text {. }
$$

Figure 4 Relative benefit in decoding success probability for varying packet sizes $(n=10,50$, 100 bits) expressed in percentage at BER of $10^{-3}$ (see online version for colours)

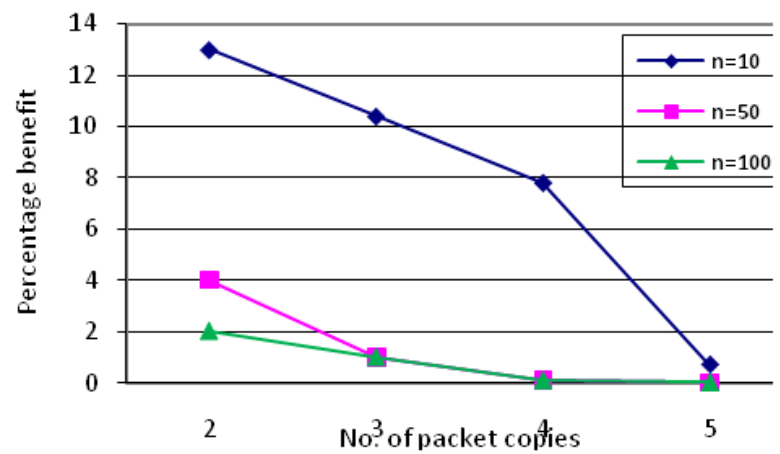


The elimination of hidden errors from the packet candidate minimises the false positives. Further, small packet is preferred since the probability of false positive reduces with packet size for any given FCS polynomial. Furthermore, there is no additional retransmission latency incurred in our proposed MAC layer mechanism for low rate RPW network.

\subsection{Prioritised communication using polarisation diversity}

In the original proposal of RPW communication system of Takei (2013), only the polarisation path with least error rate is chosen at the receiver as there is no feedback loop to adapt transmissions. Further, this reduces the achievable throughput and every data frame undergoes the same average error rate irrespective of the content or importance. However, as described in Section 3.1, the proposed polling-based MAC protocol supports half-duplex communication between the master and slave nodes that allows for path selection based on feedback. This enables the polarisation controller to include algorithm to determine the mapping of information bits to polarisation angles based on their importance or priority. Figure 5 outlines this concept.

Figure 5 Polarisation diversity transmission to achieve prioritisation

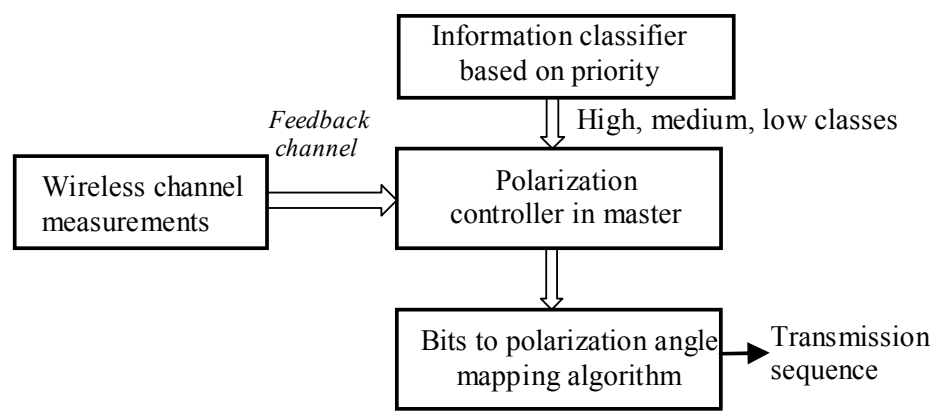

Information is classified based on tolerable error rate (TER) that is the maximum BER that can be tolerated by the content without significant distortion. For simplicity, consider three classes: high, medium and low. For reliable communication over the wireless medium, it is necessary to ensure that the $B E R \leq T E R$. Therefore, the High bits are mapped to channels with low BER, the Medium bits are mapped to channels with medium or low BER and the Low bits are mapped to channels with medium or higher BER. For this to be supported, unlike the case described in Section 3.2, each rotation of the polarisation angle would span over several bit durations. The information classifier can be applied at higher protocol layers, where bits can be classified as one or more of the following:

- Content: the source inherently possesses different error tolerance levels. Ex: video, voice and text need different protection levels.

- Semantics: nature of the information source such as safety vs. comfort applications, urgency of correct information (based on real time-ness, higher layer protocol support) can also be used to classify information into TER levels. 
- Network parameters: unidirectional transmission of sensor information is less tolerable to error due to no retransmission. Duplex networks are more tolerant to errors.

- Protocol: the control information needs higher protection than the non-critical data. Thus, control data has low TER.

The described scheme is directly applied to a single link. In an industrial scenario, to derive the full benefit of spatial and the polarisation diversity, practical algorithms are needed to derive multi-user gains.

\section{Analysis}

Two traffic classes are important in industrial applications: cyclic traffic (periodic data exchange between sensor and controllers) and acyclic traffic (spontaneous alarms). In the proposed scheme, slaves send acyclic data with priority (low TER) to the master when polled by replacing the cyclic data. This has the advantage of reducing both the mean update time and jitter. We assume the following for simplicity of analysis:

1 Alarm messages are generated according to the independent Poisson processes in $N$ slave nodes.

2 No packet drops take place on fully acknowledged MAC.

Definitions of terms used in analysis are provided in Table 1

$$
\begin{aligned}
& T_{c}=\sum_{i=1}^{2 N} B_{i} \\
& P_{\text {alarm }}=1-e^{-\lambda t_{c}} \approx \lambda t_{c} .
\end{aligned}
$$

Thus,

$$
T_{u}=\sum_{i=1}^{F} T_{c}(i), P[F=k]=\left(1-P_{\text {alarm }}\right) P_{\text {alarm }}^{k-1}
$$

where, $T_{c}(i)$ is $i^{\text {th }}$ the cycle time duration, $F$ is a geometric random variable, $k=1,2 \ldots$

From Miorandi and Vitturi (2004a), mean of the update

$$
t_{u}=\frac{t_{c}}{1-P_{\text {alarm }}}=\frac{2 N t_{\text {ack }}}{1-2 N t_{a c k} \lambda}
$$

and variance,

$$
\sigma_{T_{u}}^{2}=\frac{8 N^{3} t_{a c k}^{3} \lambda}{\left(1-2 N t_{a c k} \lambda\right)^{2}}+\frac{2 N \sigma_{T_{a c k}}^{2}}{1-2 N t_{a c k} \lambda} .
$$

The alarm latency can be expressed as

$$
d=\frac{E\left[T_{u}^{2}\right]}{2 t_{u}}+t_{a c k} .
$$

Here for each random variable $X$, we will denote its mean by $x=E[X]$, and its variance by $\sigma_{x}^{2}=E\left[X^{2}\right]-x^{2}$, where $E[$.$] denotes the statistical expectation. The mean and variance$ 
of $T_{a c k}$ in the polling-based MAC can be derived from expressions in Miorandi and Vitturi (2004a) as:

$$
\begin{aligned}
t_{\text {ack }}= & \left(T_{\text {data }}-\frac{T_{\text {slot }}}{2}\right) \cdot\left(1-6 P_{e}^{5}+5 P_{e}^{6}\right) \\
& +\left(1-P_{e}\right) T_{\text {slot }} \cdot \frac{1-\left(2 P_{e}\right)^{5}}{1-2 P_{e}}-\frac{T_{\text {slot }}}{2} \cdot\left(1-P_{e}^{5}\right) \\
\sigma_{T_{\text {ack }}}^{2} & =-t_{\text {ack }}^{2}+\left(1-P_{e}\right) \sum_{n=1}^{5} n^{2} T_{\text {data }}^{2} P_{e}^{n-1}
\end{aligned}
$$

Figure 6 (a) Mean update time variation with no. of slave nodes (N - 1 to 100) (b) Mean update time variation with alarm arrival rate $(\lambda-0.001,0.01,0.1)$ (c) Mean alarm latency variation with no. of slave nodes ( $\mathrm{N}-1$ to 100$)(\mathrm{d})$ Update time jitter variation with no. of slave nodes ( $\mathrm{N}-1$ to 100$)$ (see online version for colours)

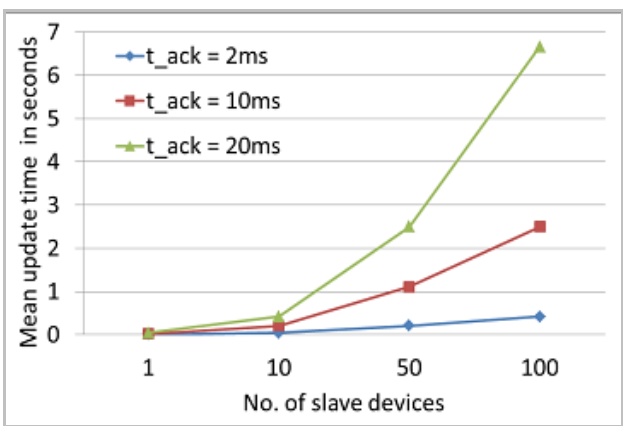

(a)

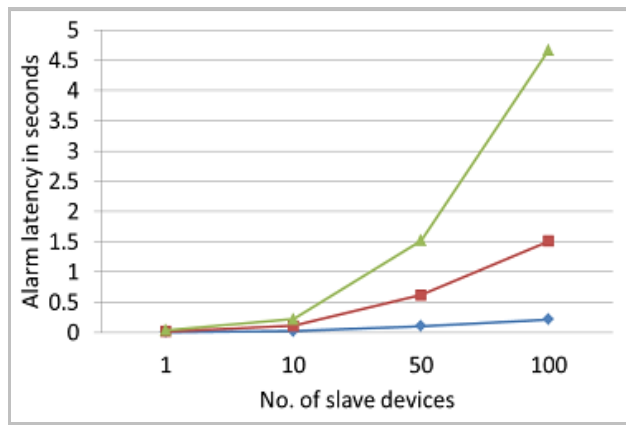

(c)

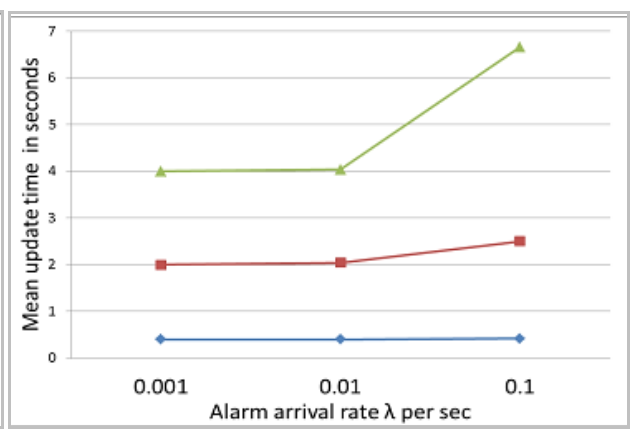

(b)

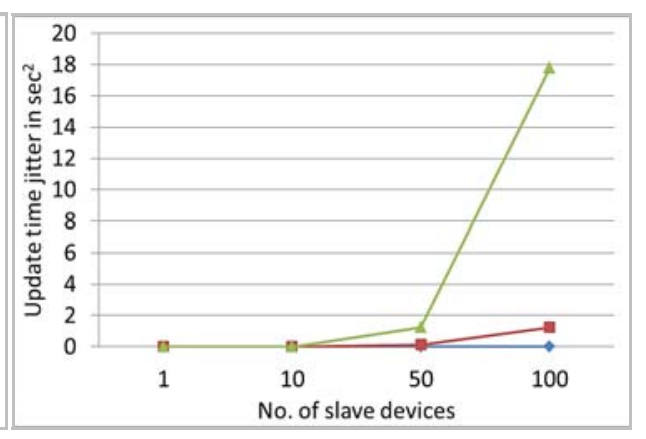

(d)

Notes: Computations at PER of $10^{-3}, t_{a c k}->2,10,20 \mathrm{~ms}, \lambda=0.1 \mathrm{~s}^{-1}$ for (a), (c), (d). Uniform packet length of 100 bits and $100 \mathrm{Kbps}$ PHY rate.

Figure 6 illustrates the impact of network parameters such as number of slave nodes and alarm generation rate on latency. To minimise update latency and jitter, it is important to keep the $T_{a c k}$ period small $(\leq 10 \mathrm{~ms})$. Also for larger networks $(\geq 50$ nodes), alarm latencies are unacceptable especially for $T_{a c k} \geq 2 \mathrm{~ms}$. This imposes restriction on the data rate $(10 \mathrm{Kbps}$ to $100 \mathrm{Kbps})$ and MAC frame size (100 bits). Through such evaluation of 
MAC protocol, the RPW network specification for industrial application can be determined.

\section{MAC layer extensions to support multihop tree topology}

For several potential industrial IoT use cases where RPW technology is suitable to be applied, a major challenge is the limited coverage of single hop network with star topology. These challenges primarily arise due to construction geometry (ex. factory floors), electromagnetic isolation (ex. Elevator shafts), transmit power limitation (ex. regional regulations for sub- $\mathrm{GHz}$ band), etc. Under the above circumstances, it is essential to support multi-hop network using RPW radio nodes. Major issues in multi-hop include topology management, interference mitigation by non-overlapping transmission and reducing end-to-end delay of the network. However, direct extensions of available multi-hop MAC protocols to RPW does not yield the required results. This is because, conventional technologies typically use either deterministic (time-slotted and fixed schedule) or probabilistic (random access) to ensure nodes do not transmit simultaneously. On the other hand, multiple simultaneous transmissions can be uniquely decoded at the RPW receiver if they consist of distinct polarisation rotation rates. Polarisation rotation rates however also determine the modulation rates of RPW transmitter. Therefore, it is essential to allocate the rotation rates to RPW nodes based on their throughput requirements and topological presence. Figure 7 shows an example of such multihop deployment in elevator scenario consisting of four hops.

Figure 7 Multihop deployment of RPW nodes in elevator tower

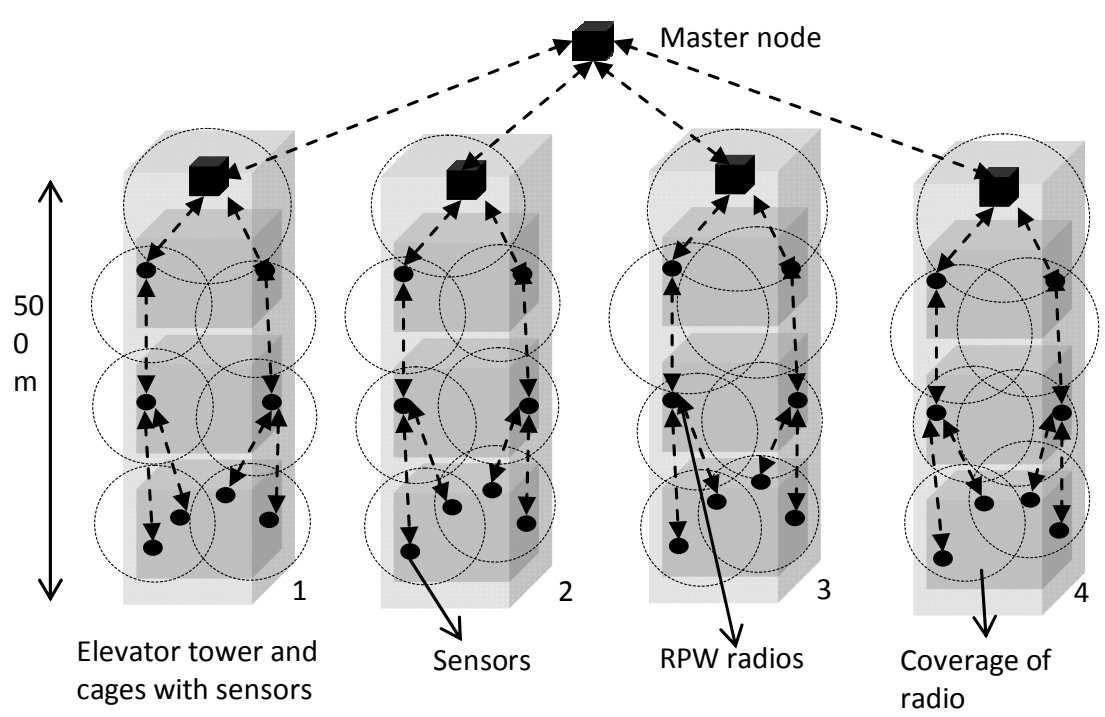

\subsection{Rotation frequency assignment to RPW nodes}

Various means are employed to assign the most suitable rotation frequency to each node in a network such as in Figure 7. A self-configuring approach assisted by a MAC layer 
management entity present at the master node is proposed. The following conditions are ensured during the assignment:

- Assignment to individual nodes should ensure that every aggregator node's rotation frequency is greater than the sum of its subordinate nodes. This is required to avoid bottleneck queues at overloaded nodes.

- The rotation frequency set is partitioned to satisfy the hierarchy requirement by appropriate mathematical method. This feature can be implemented in both centralised and distributed manner.

- The initial discovery and allocation of rotating frequency can be performed on a common well-known communication channel (rotating frequency, time slot, etc.), so that every node can discover its neighbours.

- Concurrent transmissions now possible at each level of the tree using non-overlapping rotational polarisation frequency. This reduces the required number of time slots significantly and reduces the latency.

Figure 8 shows the outline of one such method of allocation of rotation frequencies. The topology discovery step ensures each node is aware about its neighbourhood and may use Layer 1 or Layer 3 methods to identify neighbour settings. Ex: channel scanning, router advertisement and solicitation frames. Step 2 initialises a triangular matrix as elements across the main diagonal are zeroes due to uniqueness requirement. It can also be stored as a 3-D matrix with each non-zero element of the matrix stores as array $\left[\left(a_{i, j}, b_{i, j}\right)\right]$. Step 3 depends on regional regulations for available frequency band. The number of total possibilities for rotation frequencies (ex: a case of $\mathrm{N}=10$ available frequency channels considering two narrowly spaced carriers $\mathrm{f}_{1}$ and $\mathrm{f}_{2}$ each time) can be computed as

$$
\text { Combinations }=C(10,2)=\frac{n !}{(n-r) ! r !}=\frac{10 !}{(10-2) ! 2 !}=45 .
$$

Assignment search operation begins at top of the column matrix and proceeds till the last element of the column $\left[\left(a_{0, j}, b_{0, j}\right)\right]$ to $\left[\left(a_{N, j}, b_{N, j}\right)\right]$. After each allocation in each column space to nodes at a given level in tree, replace the matrix element by 0 . This avoids conflicting assignments to node in the same level of the tree. If more nodes in the same level need to be assigned $f_{r}$ after exhausting the column of matrix, then increment the column to higher $f_{r}$ and search for allocations. If all nodes at a level of the tree are allocated $f_{r}$, then increment tree level for assignment till Master node. Increment column levels to next higher column until the max. $(\mathrm{N})$ is reached. The data transmission can begin after conclusion of this stage.

\subsection{End-to-end delay analysis for multihop RPW network}

In the proposed scheme, it is clear that if one hop neighbours are assigned non-overlapping rotating frequencies, concurrent transmissions are possible. Therefore, the end-to-end latency for transmission of sensor packets to the master node should be reduced in comparison to the conventional wireless networks. In this section, we present an analytical model of end-to-end latencies comparing RPW to conventional network, 
considering a strand of the multihop network shown in Figure 7. The nodes are arranged in a tandem queuing network in Figure 9.

Figure 8 Flowchart for computation of rotating frequency at the configuration manager for tree network

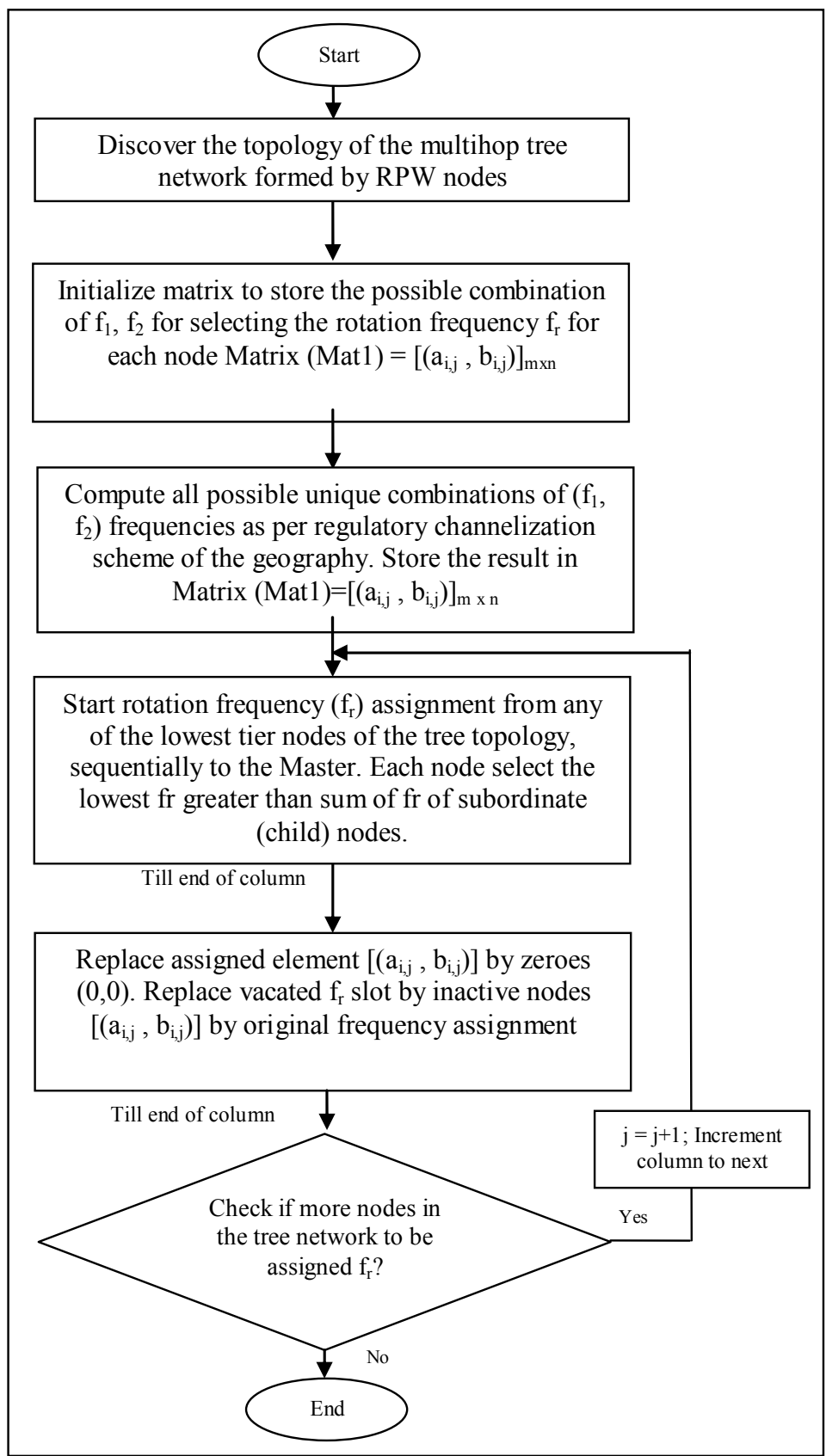


Figure 9 Network topology and sensing ranges used for analysis (see online version for colours)

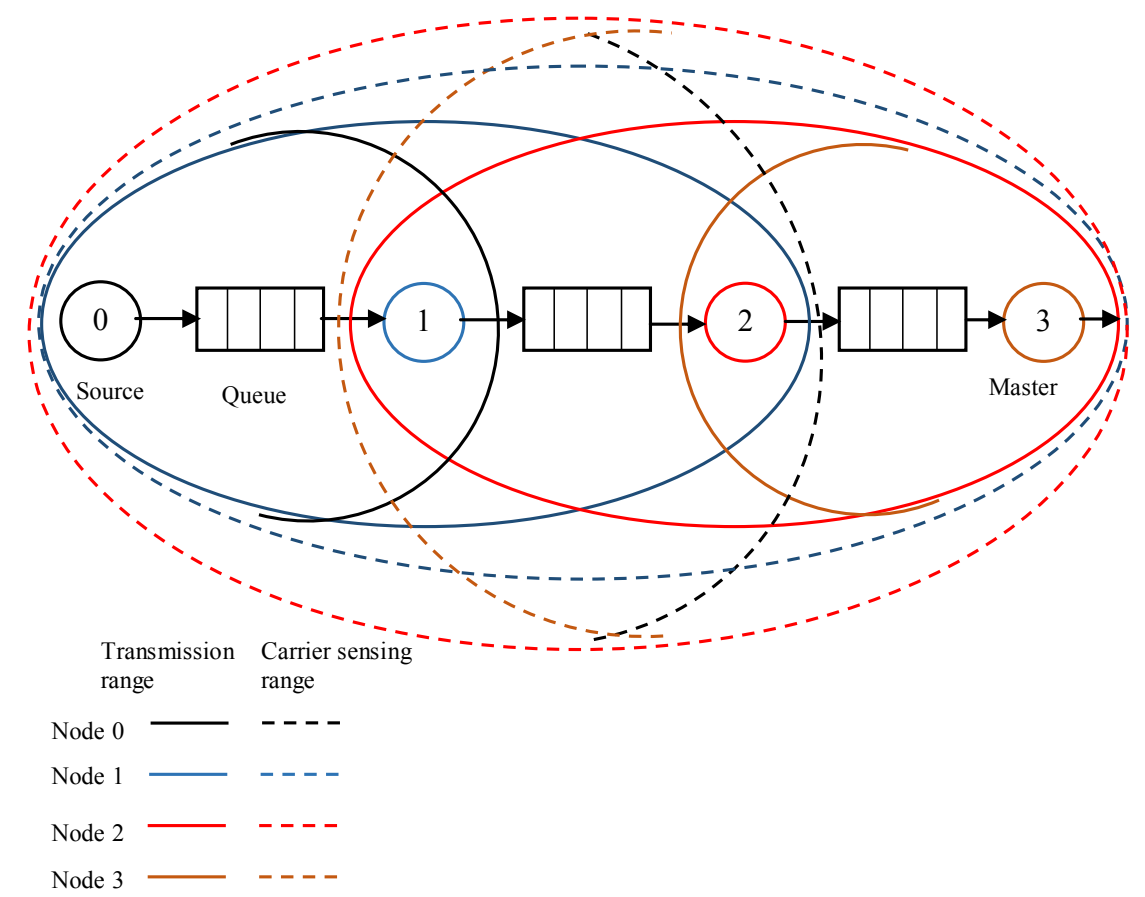

Figure 9 shows a three-hop topology similar to the network of Figure 7. However, the results derived in this section can be easily extended to a general case of $H$ hop topology. The analysis is based on the following assumptions as in Sanada et al. (2015) and Sanada and Sekiya (2017) and represents a functional non-saturated network.

1 All the network nodes use the same radio channel and equal transmission ranges are assumed for simplicity of analysis. In case of RPW 1-hop neighbours are assured to work on distinct rotating polarisation rates.

2 Only the source node generates fixed size frames following Poisson distribution, the end destination being the node $H$.

3 Channel conditions of all the links are ideal and only frame collisions are accounted for in the analysis.

4 Node $i$ can transmit frames only to node $i \pm 1$. This is termed transmission range in Figure 9. In addition, nodes $i \pm 1$ and $i \pm 2$ can sense node $i$ transmissions.

5 Each node has an infinite buffer to store frames and there assumed to suffer no packet loss at queues.

In this analysis, we use the airtime expressions that are time shares of the node states with respect to each node. The transmission airtime of node $i$ is expressed by

$$
X_{i}-\lim _{\text {time } \rightarrow \infty} \frac{s_{i}}{\text { time }}
$$


where $s_{i}$ is the sum of durations of all frame transmissions. For this analysis, we assume each of the node shares common transmission airtime given by $K$.

The carrier sensing airtime consists of frame reception durations from the previous node and carrier sensing durations from other nodes in the carrier sensing range. The carrier sensing airtime of Node $i$ is

$$
Y_{i}=\sum_{j \in v(i)}\left(X_{j}-\frac{X_{j} X_{j+3}}{1-X_{j+1} X_{j+2}}\right)
$$

where $v(i)$ is the set of carrier sensing node numbers of node $i$. For the network shown in Figure 9, the $v(i)$ of each node is given in Table 2.

Table 2 Set of carrier sensing node numbers

\begin{tabular}{lc}
\hline Node & Carrier sensing nodes \\
\hline $\mathrm{i}=0$ & $i_{1}, i_{2}$ \\
$\mathrm{i}=1$ & $i_{0}, i_{2}, i_{3}$ \\
$\mathrm{i}=2$ & $i_{0}, i_{1}, i_{3}$ \\
$\mathrm{i}=3$ & $i_{1}, i_{2}$ \\
\hline
\end{tabular}

The channel idle airtime is expressed as

$$
Z_{i}=1-X_{i}-Y_{i}=1-X_{i}-\sum_{j \in v(i)}\left(X_{j}-\frac{X_{j} X_{j+3}}{1-X_{j+1}-X_{j+2}}\right)
$$

The frame collision probability of node $i$ is expressed as

$$
\gamma_{i}=\gamma_{H_{i}}+\gamma_{C_{i}}
$$

where $\gamma_{H_{i}}$ is hidden node collision probability of node $i$ and $\gamma_{C_{i}}$ is carrier sensing nodes collision probability of node $i$.

The hidden node collision probability of node $i$ can be expressed as

$$
\gamma_{H_{i}}=\frac{a\left(X_{i+3}+X_{i}\right)}{1-X_{i+1}-X_{i+2}}
$$

where $a=\frac{D A T A}{D A T A+I F S+A C K}, D A T A$ is the transmission time of DATA frame and IFS is the interframe space and $A C K$ is the transmission time for acknowledgement.

The carrier sensing node collision probability is obtained as

$$
\gamma_{C_{i}}=1-\prod_{j \in v(i), j \neq i-2}\left(1-\tau_{j}\right)
$$

where $\tau_{i}$ is the frame transmission probability of node $i$.

For simplicity, we assume the frame transmission probability of each of the nodes to be a constant $\tau$.

In case of RPW, it assumed the nodes within the carrier sensing range allocated distinct polarisation rotation rates and hence do not interfere with each other. Therefore, $\gamma_{C_{i}}=0$ for RPW network is a feasible assumption. 
Each single hop transmission delay consists of the MAC access delay at each node and the queuing delay.

$$
D_{i}=D_{M_{i}}+D_{Q_{i}}
$$

The MAC access delay can be further computed in terms of air times as

$$
D_{M_{i}}=\frac{T R_{i}\left(X_{i}+q_{i} Z_{i}\right)}{X_{i}\left(X_{i}+Z_{i}\right)}
$$

where $T=D A T A+I F S+A C K, R_{i}=1+\gamma_{i}^{1}+\gamma_{i}^{2} \ldots+\gamma_{i}^{L}$, is the average number of transmission attempts for node $i$ and $L$ is the retransmission limit number. $q_{i}$ is the frame existence probability in idle state.

The queuing delay of node $i$ is derived in Sanada et al. (2015) to be

$$
D_{Q_{i}}=\frac{D_{M_{i}} Q_{i}\left(1+Q_{i}\right)}{2\left(1-Q_{1}\right)} .
$$

Therefore, the single hop transmission delay can be written from equation (24) as

$$
D_{i}=\frac{T R_{i} Q_{i}\left(2-Q_{i}+Q_{i}^{2}\right)}{2 X_{i}\left(1-Q_{i}\right)} .
$$

The end-to-end delay of the tandem topology depicted in Figure 9 can then be expressed as

$$
D=\sum_{i=0}^{H-1} D_{i}=\sum_{i=0}^{H-1} \frac{T R_{i} Q_{i}\left(2-Q_{i}+Q_{i}^{2}\right)}{2 X_{i}\left(1-Q_{i}\right)}
$$

\section{Numerical analysis:}

Applying equations (18) to (28) for the specific case of Figure 9, we obtain the single hop transmission delays at each node and thereby, the end-to-end delay of the wireless network.

$$
D_{0}=\frac{T R_{0} Q_{0}\left(2-Q_{0}+Q_{0}^{2}\right)}{2 X_{0}\left(1-Q_{0}\right)}
$$

where $R_{0}=\left(1+\gamma_{0}^{1}+\gamma_{0}^{2}+\gamma_{0}^{3}+\gamma_{0}^{4}\right)$ assuming retransmission limit $L=4$ and

$$
\begin{aligned}
& \gamma_{0}=\frac{2 K a}{1-2 K}+1-(1-\tau)^{2} \\
& Q_{0}=\frac{K(1-K)(1-2 K)+q_{0}\left(1-4 K+11 K^{2}-10 K^{3}\right)}{\left(1-3 K+10 K^{2}-8 K^{3}\right)} \\
& D_{1}=\frac{T R_{1} Q_{1}\left(2-Q_{1}+Q_{1}^{2}\right)}{2 X_{1}\left(1-Q_{1}\right)}
\end{aligned}
$$


where $R_{1}=\left(1+\gamma_{1}^{1}+\gamma_{1}^{2}+\gamma_{1}^{3}+\gamma_{1}^{4}\right)$ assuming retransmission limit $L=4$ and

$$
\begin{aligned}
\gamma_{1} & =\frac{K a}{1-2 K}+1-(1-\tau)^{3} \\
Q_{1} & =\frac{K-3 K^{2}+2 K^{3}+q_{1}\left(1-3 K-7 K^{3}\right)}{\left(1-2 K-K^{2}-5 K^{3}\right)} \\
D_{2} & =\frac{T R_{2} Q_{2}\left(2-Q_{2}+Q_{2}^{2}\right)}{2 X_{2}\left(1-Q_{2}\right)}
\end{aligned}
$$

where $R_{2}=\left(1+\gamma_{2}^{1}+\gamma_{2}^{2}+\gamma_{2}^{3}+\gamma_{2}^{4}\right)$ assuming retransmission limit $L=4$ and

$$
\begin{aligned}
& \gamma_{2}=\frac{K a}{1-K}+1-(1-\tau)^{3} \\
& Q_{2}=\frac{K-2 K^{2}+q_{2}\left(1-4 K+7 K^{2}\right)}{\left(1-3 K+5 K^{2}\right)} \\
& D_{3}=\frac{T R_{3} Q_{3}\left(2-Q_{3}+Q_{3}^{2}\right)}{2 X_{3}\left(1-Q_{3}\right)}
\end{aligned}
$$

where $R_{3}=\left(1+\gamma_{3}^{1}+\gamma_{3}^{2}+\gamma_{3}^{3}+\gamma_{3}^{4}\right)$ assuming retransmission limit $L=4$ and

$$
\begin{aligned}
& \gamma_{3}=K a+1-(1-\tau)^{2} \\
& Q_{3}=\frac{K(1-K)(1-2 K)+q_{0}\left(1-4 K+11 K^{2}-10 K^{3}\right)}{\left(1-3 K+10 K^{2}-8 K^{3}\right)} .
\end{aligned}
$$

Therefore, average end-to-end latency

$$
D=\sum_{i=0}^{3} D_{i}
$$

The relative performance gain in terms of the end-to-end delay by introducing RPW nodes in the multihop network can be expressed as,

$$
\text { Percentage Gain }=\frac{D_{\text {conventional network }}-D_{R P W} \text { network }}{D_{\text {conventional network }}} \times 100 .
$$

Table 3 Parameter assumption for analysis

\begin{tabular}{lc}
\hline Parameter & Values \\
\hline Transmission airtime $X_{i}=K$ & $K=0.1$ \\
$a$ assuming ACK $<<$ DATA & $a=1$ \\
$q_{i}$ frame existence probability & $q_{i}=0.1$ \\
Round trip transmission time $T$ & $T=1$ \\
Frame transmission probability $\tau_{i}$ & $\tau_{i}=0.01$ to 0.1 \\
\hline
\end{tabular}


We analyse the percentage gain by applying equations (29) to (33) assuming the following parameter values.

Figure 10 Relative improvement in end-to-end latency of multihop RPW network w.r.t conventional network (see online version for colours)

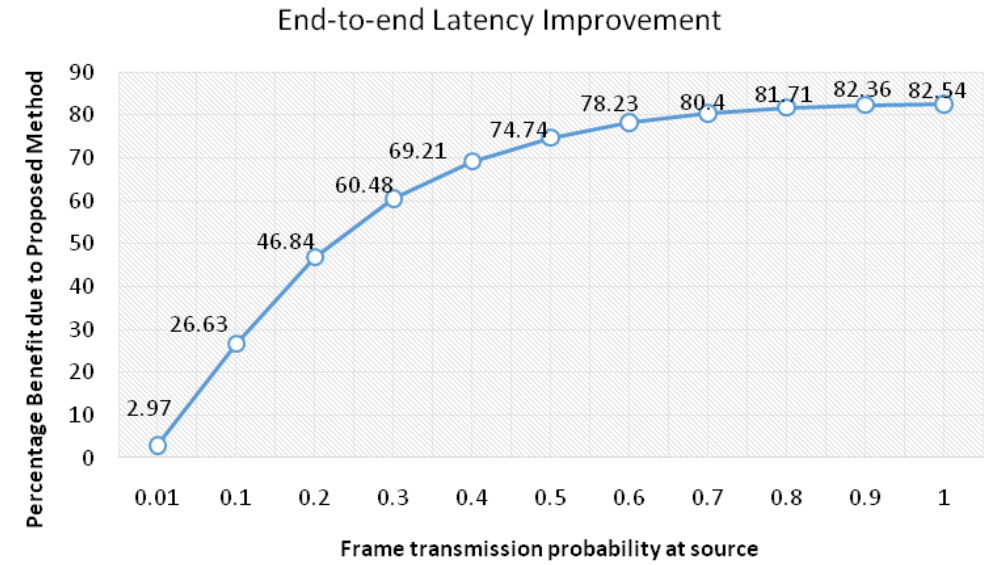

Figure 10 shows the average gain in end-to-end latency of frames traversing the multihop network of Figure 9 as a function of the frame transmission probability from the source node or sensor. The numerical results indicate that for a near-saturated network state, the end-to-end latency is reduced by up to $82 \%$. For low duty cycle sensors (transmission probability $<=0.1$ ) also, an improvement of up to $26 \%$ is achievable under evaluation conditions.

Further, the impact of scheduling concurrent transmissions in multihop network is considered by evaluating the average packet throughput over multiple links of the considered tandem network of Figure 9.

From Sanada et al. (2015), the throughput of node $i$ is given by

$$
E_{i}=X_{i}\left(1-\gamma_{i}\right) \frac{P}{T}
$$

where, $T=D A T A+I F S+A C K$

$P$ is the payload size of the $D A T A$ frame

$X_{i}$ is the transmission airtime

$\gamma_{i}$ is the collision probability.

The average throughput of the network is considered the average of individual link throughputs.

$$
E_{A v g}=\frac{\sum_{i} E_{i}}{N}
$$

Applying equations (18)-(21) and equations (34)-(35), the percentage benefit in throughput of RPW-based multihop network can be expressed as: 
Percentage Gain $=\frac{E_{A v g_{-} R P W}-E_{A v g_{-} \text {Conventional }}}{E_{A v g_{-} R P W}} \times 100$.

Figure 11 shows the results of numerical analysis of throughput for parameters shown in Table 3.

Figure 11 Relative improvement in end-to-end throughput of multihop RPW network w.r.t conventional network (see online version for colours)

\section{End-to-end Throughput Improvement}

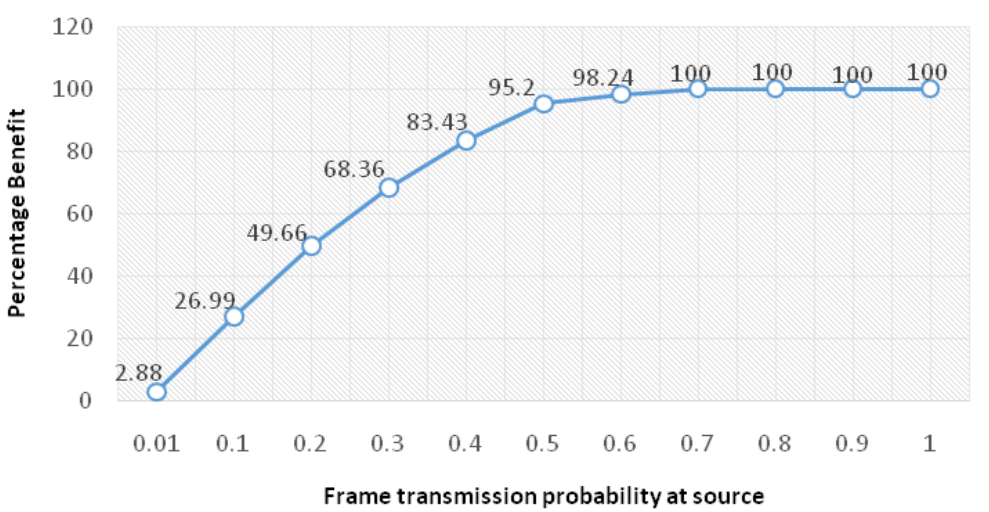

It can be observed from Figure 11 that, the benefit in network throughput monotonously increases as a function of the frame transmission probability at source node. On analysing the trend on individual links, it is found that for RPW network the throughput remains stable even with increase in number of hops, whereas in case of conventional network the throughput reduces with the increase in network load. This points to the fact that collision probability rises with increased concurrent transmissions in case of conventional networks. By ensuring non-overlapping rotation frequencies for neighbour nodes, the RPW multihop network not only reduces the end-to-end latency but also increases the average throughout of each link.

\section{Conclusions and future work}

In this paper, a reliable MAC layer scheme based on polling paradigm for RPW has been described. Link layer method to reliably combine multiple packet copies received at distinct polarisation angles is proposed. Analysis indicates that the proposed cross layer method enhances the probability of success in packet reconstruction. This is due to the key feature of the proposed method to combine frames received over independent polarisation angles at MAC layer, while simultaneously making the probability of hidden error to be infinitesimally small.

Further, means to prioritise critical information through polarisation diversity has been proposed and analytically evaluated. It is analytically proved that to minimise update latency and jitter within acceptable limits, it is important to keep the acknowledgement period small $(\leq 10 \mathrm{~ms})$. To meet these criteria for industrial 
applications, the physical layer needs to be optimised. At the lower data throughputs supported by RPW scheme, this translates to a small MAC frame size of approximately 100 bits. Non-time critical communications such as localisation of assets and goods, quality control and sensor data collection in industrial setup are some typical RPW use cases with these performance parameters.

Interference due to simultaneous packets and collision is an important challenge for multihop network. The proposed method for RPW multihop network can achieve lower end-to-end latency and therefore better throughput and quality of service when compared with conventional networks through concurrent transmissions. In near future, the MAC layer would be extended to support non-hierarchical topology such as mesh network to improve resiliency to gateway node failure issues.

\section{References}

Aono, M. and Takei, K. (2014) 'Polarization angle diversity and quick decodable block code for M2M wireless communication', in 2014 Asia-Pacific Microwave Conference, Sendai, Japan, pp.244-246.

Chakraborty, S.S., Liinabarja, M. and Yli-Juuti, E. (1999) 'An adaptive ARQ scheme with packet combining for time varying channels', in IEEE Communications Letters, Vol. 3, No. 2, pp.52-54.

Dubois-Ferrire, H., Estrin, D. and Vetterli, M. (2005) 'Packet combining in sensor networks', in Proceedings of the 3rd International Conference on Embedded Networked Sensor Systems (SenSys '05), NewYork, USA, pp.102-115.

Fujiwara, R., Miyazaki, M. and Katagishi, M. (2014) 'Low-latency wireless LAN system using polling-based MAC', in IEEE International Symposium on Circuits and Systems (ISCAS), Melbourne VIC, pp.1504-1507.

Hassan, S.M., Ibrahim, R., Bingi, K., Chung, T.D. and Saad, N. (2017) 'Application of wireless technology for control: a WirelessHART perspective', in Procedia Computer Science, Vol. 105, pp.240-247, ISSN: 1877-0509.

Liu, S., Liang, W., Zhang, X. and Zheng, M. (2014) 'Grouping TDMA access for industrial wireless sensor networks with multiple access points', Proceeding of the 11th World Congress on Intelligent Control and Automation, Shenyang, pp.146-149.

Marchenko, N., Andre, T., Brandner, G., Masood, W. and Bettstetter, C. (2014) 'An experimental study of selective cooperative relaying in industrial wireless sensor networks', in IEEE Transactions on Industrial Informatics, Vol. 10, No. 3, pp.1806-1816.

Matsuzaki, M. (2012) Reliability and Stability of Field Wireless, Yokogawa Technical Report, English Edition, Vol. 55, pp.15-18.

Miorandi, D. and Vitturi, S. (2004a) 'Analysis of master-slave protocols for real-time-industrial communications over IEEE802.11 WLANs', in INDIN '04, 2nd IEEE International Conference on Industrial Informatics, Berlin, pp.143-148.

Miorandi, D. and Vitturi, S. (2004b) 'Performance analysis of producer/consumer protocols over IEEE802.11 wireless links', in Proceedings of IEEE International Workshop on Factory Communication Systems, pp.55-64.

Nobre, M., Silva, I. and Guedes, L.A. (2015) 'Routing and scheduling algorithms for WirelessHART networks: a survey', in Sensors 2015, Vol. 15, No. 5, pp.9703-9740, DOI: 10.3390/s150509703.

O'Rourke, D. and Brennan, C. (2012) 'Practical packet combining for use with cooperative and non-cooperative ARQ schemes in resource-constrained wireless sensor networks', Ad Hoc Networks, Vol. 10, No. 3, pp.339-355. 
Perumal, T., Ganti, R.K., Koilpillai, R.D., Jalihal, D., Ramaiyan, V. and Takei, K. (2016) 'Channel estimation in rotating polarization based wireless communication systems', in Proceedings of National Conference on Communications, Guwahati, India.

Rüth, J., Schmidt, F., Serror, M., Wehrle, K. and Zimmermann, T. (2017) 'Communication and networking for the industrial internet of things', in Industrial Internet of Things, pp.317-346, Springer International Publishing.

Sanada, K. and Sekiya, H. (2017) 'Bottom-up analysis concept for throughput and delay analyses of wireless multi-hop networks', in Nonlinear Theory and Its Applications, IEICE, Vol. 8, No. 3 pp.181-203.

Sanada, K., Shi, J., Komuro, N. and Sekiya, H. (2015) 'End-to-end delay analysis for IEEE 802.11 string-topology multi-hop networks', in IEICE Transactions on Communications, No. 7, pp.1284-1293.

Takei, K. (2013) 'Polarization angle diversity for highly-reliable machine-to-machine radio', in International Conference on Circuits, Power and Computing Technologies (ICCPCT), Nagercoil.

Takei, K. (2014a) 'Balanced orthogonal code for polarization angle diversity', in 2014 Asia-Pacific Microwave Conference, Japan, pp.938-940.

Takei, K. (2014b) 'Stochastic analysis for rotating polarization communication with multi-reflection waves in multipath environment', in 2014 IEEE PES T\&D Conference and Exposition, Chicago, IL, USA, pp.1-5.

Todolí-Ferrandis, D., Silvestre-Blanes, J., Santonja-Climent, S., Sempere-Paya, V. and Vera-Pérez, J. (2017) 'Deploy\&Forget wireless sensor networks for itinerant applications', in Computer Standards \& Interfaces, ISSN: 0920-5489.

Viswanath, P., Tse, D.N.C. and Laroia, R. (2002) 'Opportunistic beamforming using dumb antennas', in Proceedings of IEEE International Symposium on Information Theory, p.449.

Wang, Q. and Jiang, J. (2016) 'Comparative examination on architecture and protocol of industrial wireless sensor network standards', in IEEE Communications Surveys \& Tutorials, Vol. 18, No. 3, pp.2197-2219. 